\title{
SUSY Predictions for and from the LHC
}

\author{
Sven Heinemeyer* \\ Instituto de Física de Cantabria (CSIC-UC), Santander, Spain \\ E-mail: Sven.Heinemeyer@cern.ch
}

On the basis of frequentist analyses of experimental constraints from electroweak precision data, $(g-2)_{\mu}, B$ physics and cosmological data, we predict the masses of Higgs bosons and SUSY particles of the CMSSM, NUHM1, VCMSSM and mSUGRA. In the CMSSM, NUHM1 and VCMSSM we find preferences for sparticle masses that are relatively light, leading to good prospects for the LHC and the ILC. Including first data from SUSY searches at the LHC leads to an increase of the preferred sparticle mass scales, but improves the consistency of the model predictions for $M_{h}$ with the LEP exclusion bounds.

Workshop on Discovery Physics at the LHC-Kruger 2010

December 05-10, 2010

Kruger National Park, Mpumalanga, South Africa

${ }^{*}$ Speaker. 


\section{Introduction}

Supersymmetry (SUSY) [1-3] is one of the favored ideas for physics beyond the Standard Model (SM) that is currently explored at the Large Hadron Collider (LHC). In several recent papers [4-8], we presented results from frequentist analyses of the parameter spaces of various GUT based versions of the Minimal Supersymmetric Standard Model (MSSM), most recently [9] also including first data from SUSY searches at the LHC $[10,11]$. We recall that the CMSSM has four input parameters: the universal soft SUSY-breaking scalar and gaugino masses $\left(m_{0}, m_{1 / 2}\right)$, a universal trilinear soft SUSY-breaking parameter $A_{0}$ and the ratio of Higgs v.e.v.'s, $\tan \beta$, as well as the sign of $\mu$ (the magnitude of $\mu$ and the bilinear SUSY-breaking parameter $B_{0}$ are fixed by the electroweak vacuum conditions). The results on the anomalous magnetic moment of the muon, $(g-2)_{\mu},[12-15]$ strongly favor a positive sign. In the NUHM1, a common soft SUSY-breaking contribution to the masses of the two Higgs doublets is allowed to vary independently, so there are five independent parameters. On the other hand, the VCMSSM imposes the supplementary constraint $B_{0}=A_{0}-m_{0}$ on the CMSSM (see Ref. [8] for our notation), thereby removing $\tan \beta$ as a free input and leaving three parameters, on which the further constraint $m_{3 / 2}=m_{0}$ in mSUGRA imposes a severe restriction. (Detailed references for the various models can be found in Ref. [9].)

Here we review the results presented in Refs. $[6,8,9]$. They include the parameters of the best-fit points in the CMSSM, NUHM1, VCMSSM and MSUGRA, as well as the 68 and 95\% CL regions for various sparticle masses applying the phenomenological, experimental and cosmological constraints. These include precision electroweak data, $(g-2)_{\mu}, B$-physics observables (the rates for $\mathrm{BR}(b \rightarrow s \gamma)$ and $\mathrm{BR}\left(B_{u} \rightarrow \tau \nu_{\tau}\right), B_{s}$ mixing, and the upper limit on $\left.\mathrm{BR}\left(B_{s} \rightarrow \mu^{+} \mu^{-}\right)\right)$, the bound on the lightest MSSM Higgs boson mass, $M_{h}$, and the cold dark matter (CDM) density inferred from astrophysical and cosmological data, assuming that this is dominated by the relic density of the lightest neutralino, $\Omega_{\chi} h^{2}$. In the case of Ref. [9] also recent limits from SUSY searches at CMS and ATLAS $[10,11]$ are taken into account.

\section{Description of our Frequentist approach}

We define a global $\chi^{2}$ likelihood function, which combines all theoretical predictions with experimental constraints (except the latest LHC SUSY searches):

$$
\begin{aligned}
\chi^{2}\left(\equiv \chi_{\mathrm{org}}^{2}\right) & =\sum_{i}^{N} \frac{\left(C_{i}-P_{i}\right)^{2}}{\sigma\left(C_{i}\right)^{2}+\sigma\left(P_{i}\right)^{2}}+\sum_{i}^{M} \frac{\left(f_{\mathrm{SM}_{i}}^{\mathrm{obs}}-f_{\mathrm{SM}_{i}}^{\mathrm{fit}}\right)^{2}}{\sigma\left(f_{\mathrm{SM}_{i}}\right)^{2}} \\
& +\chi^{2}\left(M_{h}\right)+\chi^{2}\left(\mathrm{BR}\left(B_{s} \rightarrow \mu \mu\right)\right) \\
& \left.+\chi^{2} \text { (SUSY search limits from LEP and Tevatron }\right)
\end{aligned}
$$

Here $N$ is the number of observables studied, $C_{i}$ represents an experimentally measured value (constraint) and each $P_{i}$ defines a prediction for the corresponding constraint that depends on the supersymmetric parameters. The experimental uncertainty, $\sigma\left(C_{i}\right)$, of each measurement is taken to be both statistically and systematically independent of the corresponding theoretical uncertainty, $\sigma\left(P_{i}\right)$, in its prediction. We denote by $\chi^{2}\left(M_{h}\right)$ and $\chi^{2}\left(\operatorname{BR}\left(B_{s} \rightarrow \mu \mu\right)\right)$ the $\chi^{2}$ contributions from the two measurements for which only one-sided bounds are available so far, as discussed below. 
Furthermore we include the lower limits from the direct searches for SUSY particles at LEP [16] as one-sided limits, denoted by " $\chi^{2}$ (SUSY search limits)" in eq. (2.1).

We stress that in $[6,8,9]$ (as in $[4,5,7])$ the three SM parameters $f_{\mathrm{SM}}=\left\{\Delta \alpha_{\text {had }}, m_{t}, M_{Z}\right\}$ are included as fit parameters and allowed to vary with their current experimental resolutions $\sigma\left(f_{\mathrm{SM}}\right)$. We do not include $\alpha_{s}$ as a fit parameter, which would have only a minor impact on the analysis.

Formulating the fit in this fashion has the advantage that the $\chi^{2}$ probability, $P\left(\chi^{2}, N_{\mathrm{dof}}\right)$, properly accounts for the number of degrees of freedom, $N_{\text {dof }}$, in the fit and thus represents a quantitative and meaningful measure for the "goodness-of-fit." In previous studies [4], $P\left(\chi^{2}, N_{\text {dof }}\right)$ has been verified to have a flat distribution, thus yielding a reliable estimate of the confidence level for any particular point in parameter space. Furthermore, an important aspect of the formulation is that all model parameters are varied simultaneously in our MCMC sampling, and care is exercised to fully explore the multi-dimensional space, including possible interdependencies between parameters. All confidence levels for selected model parameters are performed by scanning over the desired parameters while minimizing the $\chi^{2}$ function with respect to all other model parameters. The function values where $\chi^{2}(x)$ is found to be equal to $\chi_{\min }^{2}+\Delta \chi^{2}$ determine the confidence level contour. For two-dimensional parameter scans we use $\Delta \chi^{2}=2.23(5.99)$ to determine the $68 \%(95 \%)$ confidence level contours. Only experimental constraints are imposed when deriving confidence level contours, without any arbitrary or direct constraints placed on model parameters themselves. This leads to robust and statistically meaningful estimates of the total $68 \%$ and $95 \%$ confidence levels, which may be composed of multiple separated contours.

The experimental constraints used in our analyses are listed in Table 1 in [6]. The only significant changes are an updated value of the top quark mass, $m_{t}^{\exp }=173.3 \pm 1.1 \mathrm{GeV}$ [17] and the use of the new $e^{+} e^{-}$determination of the SM contribution to $(g-2)_{\mu}[14], a_{\mu}^{\mathrm{SUSY}}=$ $(28.7 \pm 8.0) \times 10^{-10}$, see also Ref. [15].

One important comment concerns our implementation of the LEP constraint on $M_{h}$. The value quoted in Table 1 of Ref. [6], $M_{H}>114.4 \mathrm{GeV}$, was derived within the SM [18], and is applicable to the CMSSM, VCMSSM and mSUGRA, in which the relevant Higgs couplings are very similar to those in the SM [19,20], so that the SM exclusion results can be used, supplemented with an additional theoretical uncertainty: we evaluate the $\chi^{2}\left(M_{h}\right)$ contribution within the CMSSM, VCMSSM and mSUGRA using the formula

$$
\chi^{2}\left(M_{h}\right)=\frac{\left(M_{h}-M_{h}^{\text {limit }}\right)^{2}}{(1.1 \mathrm{GeV})^{2}+(1.5 \mathrm{GeV})^{2}},
$$

with $M_{h}^{\text {limit }}=115.0 \mathrm{GeV}$ for $M_{h}<115.0 \mathrm{GeV}$. Larger masses do not receive a $\chi^{2}\left(M_{h}\right)$ contribution. We use $115.0 \mathrm{GeV}$ so as to incorporate a conservative consideration of experimental systematic effects. The $1.5 \mathrm{GeV}$ in the denominator corresponds to a convolution of the likelihood function with a Gaussian function, $\tilde{\Phi}_{1.5}(x)$, normalized to unity and centered around $M_{h}$, whose width is $1.5 \mathrm{GeV}$, representing the theory uncertainty on $M_{h}$ [21]. In this way, a theoretical uncertainty of up to $3 \mathrm{GeV}$ is assigned for $\sim 95 \%$ of all $M_{h}$ values corresponding to one parameter point. The $1.1 \mathrm{GeV}$ term in the denominator corresponds to a parameterization of the $C L_{s}$ curve given in the final SM LEP Higgs result [18].

Within the NUHM1 the situation is somewhat more involved, since, for instance, a strong suppression of the ZZh coupling can occur, invalidating the SM exclusion bounds. In order to find 
a more reliable 95\% CL exclusion limit for $M_{h}$ in the case that the SM limit cannot be applied, we use the following procedure. The main exclusion bound from LEP searches comes from the channel $e^{+} e^{-} \rightarrow Z H, H \rightarrow b \bar{b}$. The Higgs boson mass limit in this channel is given as a function of the $Z Z H$ coupling in [22]. A reduction in the ZZh coupling in the NUHM1 relative to its SM value can be translated into a lower limit on the lightest NUHM1 Higgs mass, $M_{h}^{\text {limit, } 0}$, shifted to lower values with respect to the SM limit of $114.4 \mathrm{GeV}$. (The actual number is obtained using the code HiggsBounds [23] that incorporates the LEP (and Tevatron) limits on neutral Higgs boson searches.) For values of $M_{h} \lesssim 86 \mathrm{GeV}$ the reduction of the $Z Z h$ couplings required to evade the LEP bounds becomes very strong, and we add a brick-wall contribution to the $\chi^{2}$ function below this value (which has no influence on our results). Finally, eq. (2.2) is used with $M_{h}^{\text {limit }}=$ $M_{h}^{\text {limit, } 0}+0.6 \mathrm{GeV}$ to ensure a smooth transition to the SM case, see [6] for more details.

To include the recent LHC searches for SUSY we calculate [9]

$$
\begin{aligned}
\chi_{\text {ATLAS }}^{2} & =\chi_{\text {org }}^{2}+\Delta \chi_{\text {ATLAS }}^{2}, \\
\chi_{\text {CMS }}^{2} & =\chi_{\text {org }}^{2}+\Delta \chi_{\text {CMS }}^{2},
\end{aligned}
$$

i.e. we investigate the impact of the SUSY searches at ATLAS and CMS separately and do not attempt a combination of these searches.

The CMS result [10] is based on a search for multijet $+Z_{T}$ events without accompanying leptons. The 13 events found in the signal region were compatible with the $\sim 10.5$ expected from SM backgrounds with a probability value of $30 \%$ The observed result allowed CMS to set a 95\% CL (i.e., $1.96 \sigma$ ) upper limit of 13.4 signal events. This would correspond to $2.5 \pm(13.4-2.5) / 1.96=$ $2.5 \pm 5.6$ events for any possible signal, yielding $\chi_{\infty, \mathrm{CMS}}^{2}=0.85$ for large sparticle masses. The central CMS result, shown in Fig. 5 of [10], is a 95\% CL exclusion contour in the $\left(m_{0}, m_{1 / 2}\right)$ plane of the CMSSM for the particular values $\tan \beta=3, A_{0}=0$ and $\mu>0$. However, the sensitivity of a search for multijet $+H_{T}$ events is largely independent of these additional parameters within the CMSSM [10], and can also be taken over to the NUHM1, VCMSSM and mSUGRA models, which have similar signatures in these search channels.

Fig. 5 of [10] also presents a $\left(m_{0}, m_{1 / 2}\right)$ contour for the $95 \% \mathrm{CL}$ exclusion expected in the absence of any signal, corresponding to $5.56 \times 1.96=10.9$ events. This contour would correspond to an apparent significance of $(10.9-2.5) / 5.56 \sim 1.5 \sigma$ and hence $\Delta \chi^{2} \sim 4$. The observed $95 \%$ $\mathrm{CL}$ contour, on the other hand, corresponds to $\Delta \chi^{2}=5.99$. We approximate the impact of the new CMS constraint by $\Delta \chi_{\mathrm{CMS}}^{2} \sim \chi_{\infty, \mathrm{CMS}}^{2}\left|\left(M_{C} / M\right)-1\right|^{-p_{C}}$ (where $M \equiv \sqrt{m_{0}^{2}+m_{1 / 2}^{2}}$ ) for each ray in the $\left(m_{0}, m_{1 / 2}\right)$ plane, fitting the parameters $M_{C}, p_{C}$ by requiring $\Delta \chi^{2} \sim 4,5.99$ on the expected and observed 95\% exclusion contours shown in Fig. 5 of [10], see Ref. [9] for details.

The ATLAS result [11] is based on a search for multijet $+Z_{T}$ events with one accompanying electron or muon. The 2 events found in the signal region were compatible with the $\sim 4.1$ expected from SM backgrounds with a probability value of $16 \%$. The central ATLAS result, shown in Fig. 2 of [11], is again a 95\% CL exclusion contour in the $\left(m_{0}, m_{1 / 2}\right)$ plane of the CMSSM for the particular values $\tan \beta=3, A_{0}=0$ and $\mu>0$, which is also only moderately dependent on these additional parameters within the CMSSM [11], and can also be taken over to the NUHM1, VCMSSM and mSUGRA models. We construct $\Delta \chi_{\text {ATLAS }}^{2}$ in a similar fashion as for CMS, where all the details can be found in Ref. [9]. 
The numerical evaluation of the frequentist likelihood function using the constraints has been performed with the MasterCode [4-9,24], which includes the following theoretical codes. For the RGE running of the soft SUSY-breaking parameters, it uses SoftSUSY [25], which is combined consistently with the codes used for the various low-energy observables. At the electroweak scale we have included various codes: FeynHiggs [21,26-28] is used for the evaluation of the Higgs masses and $a_{\mu}^{\text {SUSY }}$ (see also [29-32]). For flavor-related observables we use SuF la [33, 34] as well as SuperIso [35,36], and for the electroweak precision data we have included a code based on [37,38]. Finally, for dark-matter-related observables, MicrOMEGAs [39-41] and DarkSUSY $[42,43]$ have been used. We made extensive use of the SUSY Les Houches Accord $[44,45]$ in the combination of the various codes within the MasterCode.

\section{Results for Sparticle Masses (without LHC data)}

The best-fit points and respective probabilities are summarized in Tab. 1 in Sect. 5. Here we review the results for the predictions of sparticles masses in the CMSSM, NUHM1, VCMSSM and mSUGRA. The results for the CMSSM spectrum are shown in the upper plot, and for the NUHM1 in the lower plot of Fig. 1, whereas the spectra in the VCMSSM can be found in top plot and mSUGRA in the middle and bottom plot of Fig. 2. The first (middle) mSUGRA plot corresponds to the overall minimum in $\chi^{2} \sim 29$ in this model. However, a second local minimum of $\chi^{2} \sim 33$ (and hence a "small" area allowed at the 95\% CL) can be found along the light Higgs rapid-annihilation strip with small $m_{1 / 2}$ are very large $m_{0}$. The masses shown in the second (lower) plot correspond to this secondary minimum.

We start our discussion with the gluino mass, $m_{\tilde{g}}$. In both the CMSSM and the NUHM1, the best-fit points have relatively low values of $m_{\tilde{g}} \sim 750$ and $\sim 600 \mathrm{GeV}$, respectively. These favored values are well within the range even of the early operations of the LHC with reduced center-ofmass energy and limited luminosity. However, even quite large values of $m_{\tilde{g}} \lesssim 2.5 \mathrm{TeV}$ are allowed at the $3 \sigma\left(\Delta \chi^{2}=9\right)$ level (not shown in Fig. 1). The LHC should be able to discover a gluino with $m_{\tilde{g}} \sim 2.5 \mathrm{TeV}$ with $100 / \mathrm{fb}$ of integrated luminosity at $\sqrt{s}=14 \mathrm{TeV}$ [46,47], and the proposed sLHC luminosity upgrade to $1000 / \mathrm{fb}$ of integrated luminosity at $\sqrt{s}=14 \mathrm{TeV}$ should permit the discovery of a gluino with $m_{\tilde{g}} \sim 3 \mathrm{TeV}$ [48]. However, Fig. 1 does demonstrate that, whilst there are good prospects for discovering SUSY in early LHC running [5], this cannot be 'guaranteed'.

The central values of the masses of the supersymmetric partners of the $u, d, s, c, b$ quarks are slightly lighter than the gluino, as seen in Fig. 1. The difference between the gluino and the squark masses is sensitive primarily to $m_{0}$. The reason is that the preferred regions of the parameter space in both the CMSSM and the NUHM1 are in the $\tilde{\chi}_{1}^{0}$-slepton coannihilation region $[5,6]$ where $m_{0}<m_{1 / 2}$. Here $m_{0}$ makes only small contributions to the central values of the squark masses. The SUSY partners of the left-handed components of the four lightest quarks, the $\tilde{q}_{L}$, are predicted to be slightly heavier than the corresponding right-handed squarks, $\tilde{q}_{R}$, as seen by comparing the mass ranges in Fig. 1. As in the case of the gluino, squark masses up to $\sim 2.5 \mathrm{TeV}$ are allowed at the $3 \sigma$ level. Comparing the upper and lower panels, we see that the squarks are predicted to be somewhat lighter in the NUHM1 than in the CMSSM, but this difference is small compared with the widths of the corresponding likelihood functions. 

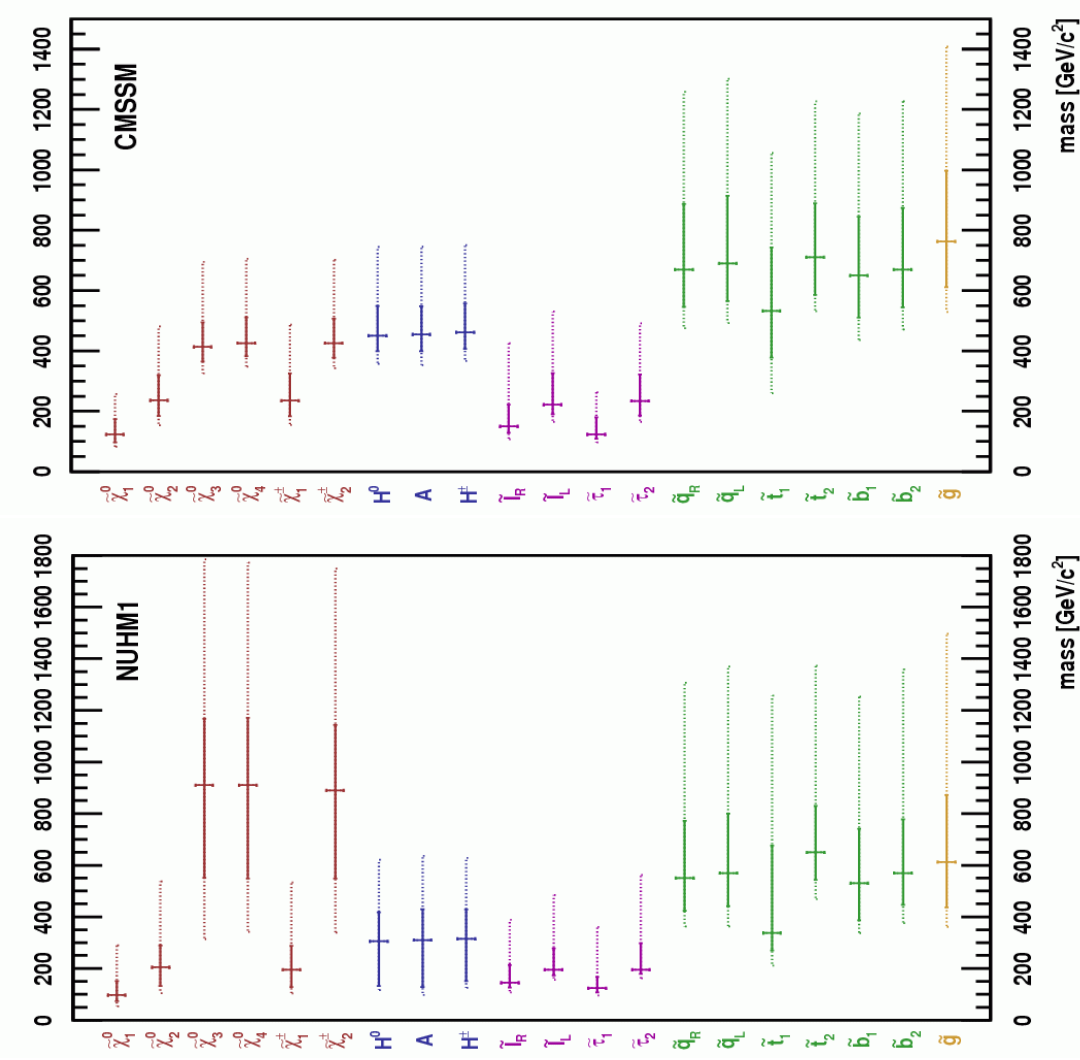

Figure 1: Spectra in the CMSSM (upper plot) and the NUHM1 (lower plot) [6]. The horizontal solid lines indicate the best-fit values, the vertical solid lines are the $68 \%$ CL ranges, and the vertical dashed lines are the $95 \%$ CL ranges for the indicated mass parameters.

Turning now to the likelihood functions for the mass of the lighter stop, $m_{\tilde{t}_{1}}$, we find that it is shifted to values somewhat lower than for the other squark flavors. It can also be seen that the $2 \sigma$ range of its likelihood function differ from those of the gluino and the other squarks, reflecting the importance of scalar top mixing. We recall that this depends strongly on the trilinear soft SUSY-breaking parameter $A_{t}$ and the Higgs mixing parameter $\mu$, as well as on the precise value of $m_{t}$.

In the case of the lighter stau $\tilde{\tau}_{1}$, see its range in Fig. 1, the mass is very similar to that of the LSP $\tilde{\chi}_{1}^{0}$ in the coannihilation region, but this is not the case in the rapid-annihilation $H, A$ funnel region, see [6] for details. In the case of the NUHM1 rapid annihilation is possible also for low $\tan \beta$, leading to larger values of $m_{0}$ than in the CMSSM also for relatively small values of $m_{\tilde{\tau}_{1}}$.

The scalar taus as well as the other scalar leptons are expected to be relatively light, as can be seen in Fig. 1. They would partially be in the reach of the ILC(500) (i.e. with $\sqrt{s}=500 \mathrm{GeV}$ ) and at the 95\% CL nearly all be in the reach of the ILC(1000) $[49,50]$. This also holds for the two lighter neutralinos and the light chargino.

In the case of the VCMSSM, the spectrum is qualitatively similar to those in the CMSSM and NUHM1. The two mSUGRA spectra are significantly different from each other and from 

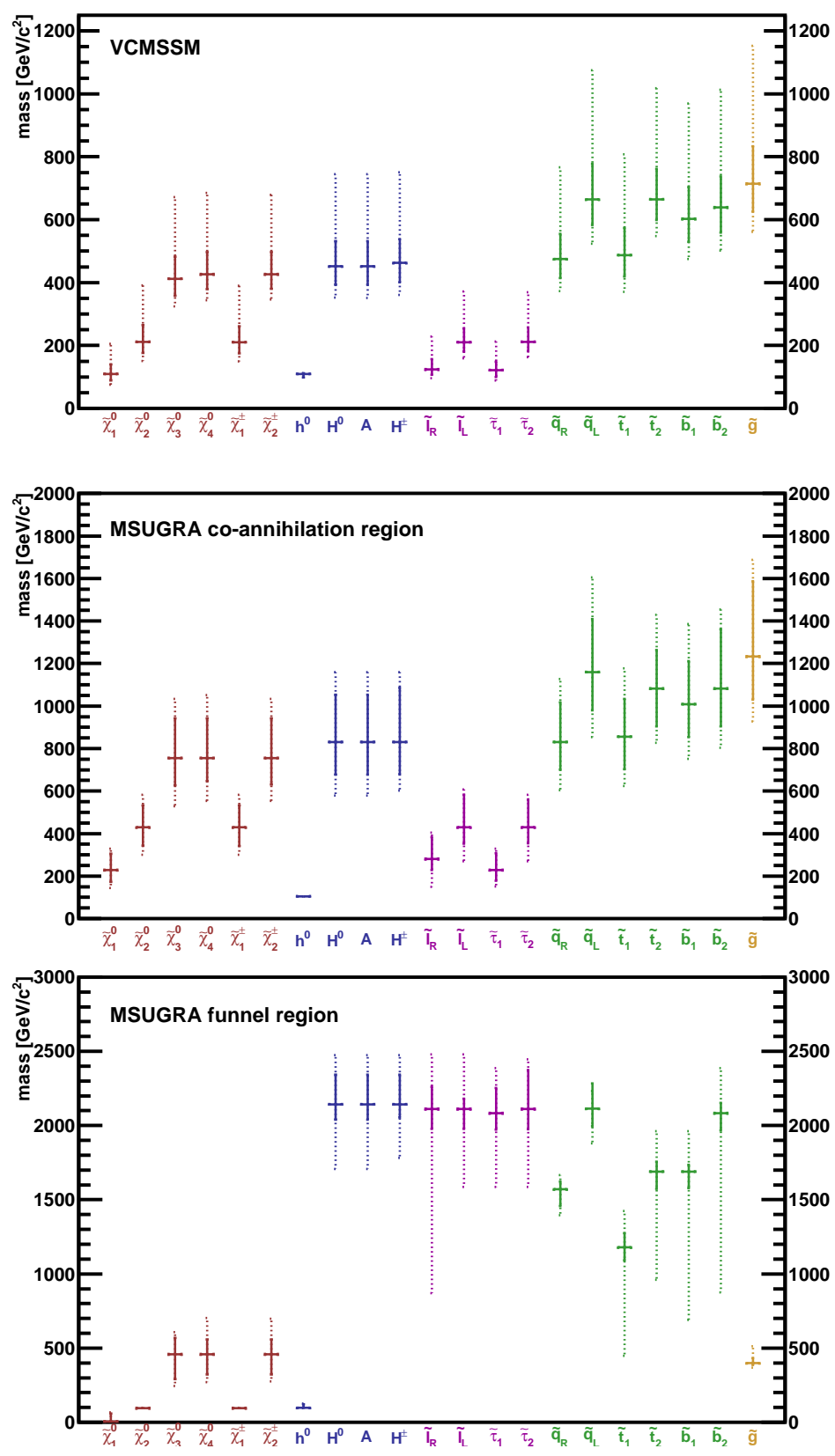

Figure 2: Spectra in the VCMSSM (top), and mSUGRA in the coannihilation region (middle) and the funnel region (bottom), implementing all the constraints including that on $\Omega_{\chi} h^{2}$. The horizontal solid lines indicate the best-fit values, the vertical solid lines are the $68 \%$ CL ranges, and the vertical dashed lines are the $95 \%$ $C L$ ranges for the indicated mass parameters.

the VCMSSM, CMSSM and NUHM1. This is because the coannihilation region has $m_{1 / 2}$ significantly larger than in the other models, whereas the funnel region has a significantly smaller and very well-defined value of $m_{1 / 2}$ and relatively large values of $m_{0}$. This bimodality affects directly the preferred values of $m_{\tilde{\chi}_{1}^{0}}$ and $m_{\tilde{g}}$, and affects the other sparticle masses via renormalization ef- 
fects. These spectra show that the colored particles are well within the reach of the LHC for the VCMSSM and mSUGRA in the coannihilation region, whereas more integrated luminosity would be necessary for mSUGRA in the funnel region (except for gluino production). In each scenario some SUSY particles should be accessible at an $e^{+} e^{-}$collider, even with a center-of-mass energy as low as $500 \mathrm{GeV}$.

\section{Prediction for the Higgs sector (without LHC data)}

In Fig. 3 we display the favored regions in the $\left(M_{A}, \tan \beta\right)$ planes for the CMSSM and NUHM1 (taken from Ref. [6]). (Predictions for $M_{h}$ in the four models are reviewed in Sect. 6.) We see that they are broadly similar, with little correlation between the two parameters. Concerning $\tan \beta$, one can observe that while the best fit values lie at $\tan \beta \approx 11$, the 68 (95)\% CL areas reach up to $\tan \beta \approx 30$ (50-60). The existing heavy neutral Higgs discovery analyses (performed in the various benchmark scenarios [51-54]) cannot directly be applied to the $\left(M_{A}, \tan \beta\right)$ planes in Fig. 3. In order to assess the prospects for discovering heavy Higgs bosons at the LHC in this context, we follow the analysis in [55], which assumed 30 or $60 \mathrm{fb}^{-1}$ collected with the CMS detector. For evaluating the Higgs-sector observables including higher-order corrections we use the soft SUSYbreaking parameters of the best-fit points in the CMSSM and the NUHM1, respectively. We show in Fig. 3 the $5 \sigma$ discovery contours for the three decay channels $H, A \rightarrow \tau^{+} \tau^{-} \rightarrow$ jets (solid lines), jet $+\mu$ (dashed lines) and jet $+\mathrm{e}$ (dotted lines). The parameter regions above and to the left of the curves are within reach of the LHC with about $30 \mathrm{fb}^{-1}$ of integrated luminosity. We see that most of the highest-CL regions lie beyond this reach, particularly in the CMSSM. At the ILC(1000) masses up to $M_{A} \lesssim 500 \mathrm{GeV}$ can be probed. Within the CMSSM this includes the best-fit point, and within the NUHM1 nearly the whole $68 \%$ CL area can be covered.
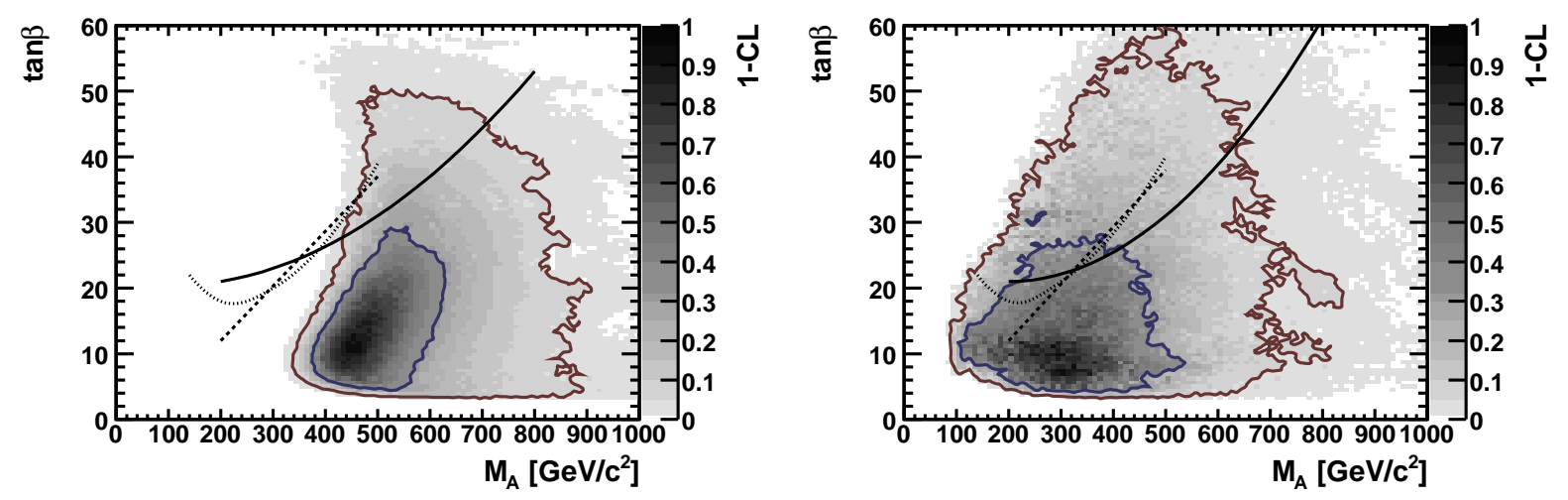

Figure 3: The correlations between $M_{A}$ and $\tan \beta$ in the CMSSM (left panel) and in the NUHM1 (right panel) [6]. Also shown are the $5 \sigma$ discovery contours for observing the heavy MSSM Higgs bosons $H$, A in the three decay channels $H, A \rightarrow \tau^{+} \tau^{-} \rightarrow$ jets (solid line), jet $+\mu$ (dashed line), jet $+\mathrm{e}$ (dotted line) at the LHC. The discovery contours have been obtained using an analysis that assumed 30 or $60 \mathrm{fb}^{-1}$ collected with the CMS detector [47, 55]. 


\section{Results including LHC data}

In this section we review the results obtained including the recently published SUSY search limits from CMS [10] and ATLAS [11] (taken from Ref. [9]). ${ }^{1}$ In Tab. 1 we compare the best-fit points found incorporating the CMS or ATLAS constraints [9] with pre-LHC results $[5,6,8]$ in the four scenarios. In addition to the minimum value of $\chi^{2}$ and the fit probability in each scenario, we include the values of $m_{1 / 2}, m_{0}, A_{0}$ and $\tan \beta$ at all the best-fit points, as well as $M_{h}$ (discarding the LEP limits). ${ }^{2}$

\begin{tabular}{|c||c|c|c|c|c|c||c|}
\hline Model & Minimum $\chi^{2}$ & Probability & $\begin{array}{c}m_{1 / 2} \\
(\mathrm{GeV})\end{array}$ & $\begin{array}{c}m_{0} \\
(\mathrm{GeV})\end{array}$ & $\begin{array}{c}A_{0} \\
(\mathrm{GeV})\end{array}$ & $\tan \beta$ & $\begin{array}{c}M_{h} \text { (no LEP) } \\
(\mathrm{GeV})\end{array}$ \\
\hline \hline CMSSM & $(21.3)$ & $(32 \%)$ & $(320)$ & $(60)$ & $(-170)$ & $(11)$ & $(107.9)$ \\
with CMS & 22.0 & $29 \%$ & 370 & 80 & -340 & 14 & 112.6 \\
with ATLAS & 24.9 & $16 \%$ & 400 & 100 & -430 & 16 & 112.8 \\
\hline NUHM1 & $(19.3)$ & $(31 \%)$ & $(260)$ & $(110)$ & $(1010)$ & $(8)$ & $(121.9)$ \\
with CMS & 20.9 & $28 \%$ & 380 & 90 & 70 & 14 & 113.5 \\
with ATLAS & 23.3 & $18 \%$ & 490 & 110 & -630 & 25 & 116.5 \\
\hline VCMSSM & $(22.5)$ & $(31 \%)$ & $(300)$ & $(60)$ & $(30)$ & $(9)$ & $(109.3)$ \\
with CMS & 23.8 & $25 \%$ & 340 & 70 & 50 & 9 & 115.5 \\
with ATLAS & 27.1 & $13 \%$ & 390 & 90 & 70 & 11 & 117.0 \\
\hline mSUGRA & $(29.4)$ & $(6.1 \%)$ & $(550)$ & $(230)$ & $(430)$ & $(28)$ & $(107.8)$ \\
with CMS & 29.4 & $6.1 \%$ & 550 & 230 & 430 & 28 & 121.2 \\
with ATLAS & 30.9 & $5.7 \%$ & 550 & 230 & 430 & 28 & 121.2 \\
\hline
\end{tabular}

Table 1: Comparison of the best-fit points found previously in the CMSSM, the NUHM1, the VCMSSM and the coannihilation region of $M S U G R A$ when the LHC constraints were not included (in parentheses) [5, 6, 8], and the results of this paper incorporating the CMS [10] and ATLAS [11] constraints [9]. In addition to the minimum value of $\chi^{2}$ and the fit probability in each scenario, we include the values of $m_{1 / 2}, m_{0}, A_{0}$ and $\tan \beta$ at all the best-fit points, as well as the predictions for $M_{h}$ neglecting the LEP constraint.

The absence of a supersymmetric signal in the LHC data $[10,11]$ invalidates portions of the CMSSM, NUHM1 and VCMSSM parameter spaces at low $m_{1 / 2}$ that were previously favored at the 95\% and 68\% CL, but does not impinge significantly on the corresponding regions for mSUGRA. In the cases of the CMSSM and VCMSSM, the LHC data disfavor the low- $m_{1 / 2}$ tips of the coannihilation regions and increase visibly the best-fit values of $m_{1 / 2}$, as seen in Fig. 4 (and in Tab. 1). However, it should be kept in mind that the area around the best-fit points is very shallow in $\chi^{2}$, particularly as a function of $m_{1 / 2}$. In the case of the NUHM1, the CMS or ATLAS data disfavor

\footnotetext{
${ }^{1}$ Anticipated results including early LHC data in the context of frequentist fits were shown, e.g., in Refs. [56, 57].

${ }^{2}$ In Ref. [9] a systematic error of $\sim 10(20) \%$ was estimated in the values for $m_{1 / 2}$ quoted in Table 1 at the best-fit points, associated with ambiguity in the implementation of the CMS (ATLAS) constraint and the slow variations in the $\chi^{2}$ functions.
} 
a slice of parameter space at low $m_{1 / 2}$ and $m_{0}<400 \mathrm{GeV}$ extending between the coannihilation region and the light-Higgs funnel discussed in [8]. However, as noted in Table 1, the new LHC constraints do not increase significantly the value of the global $\chi^{2}$ function for the previous best-fit points in any of the models. Applying the CMS constraint we find an increase of $\Delta \chi^{2} \sim 1$. The ATLAS constraint leads to somewhat stronger increase up to $\Delta \chi^{2} \sim 4.5$. This indicates that there is no significant tension between the CMS and previous constraints in the contexts of the models studied here, and only a mild tension with the new ATLAS limits. Absence of a SUSY signal at the LHC with a luminosity of $\geq 1 / \mathrm{fb}$ at a center-of-mass energy $\geq 7 \mathrm{TeV}$ would be required to increase the global minimum of $\chi^{2}$ sufficiently to put pressure on these models.
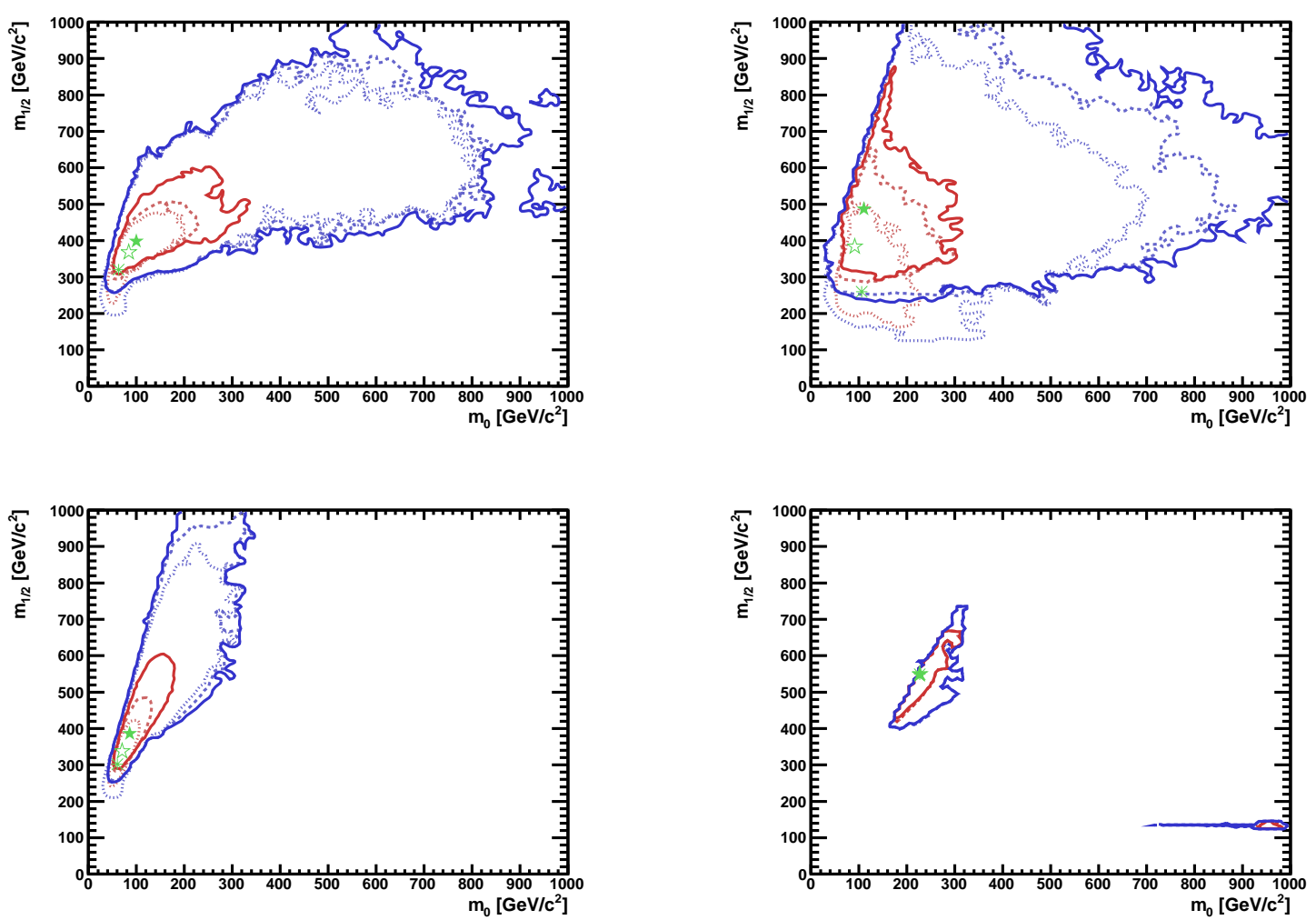

Figure 4: The $\left(m_{0}, m_{1 / 2}\right)$ planes in the CMSSM (upper left), NUHM1 (top right), VCMSSM (lower left) and mSUGRA (lower right). In each panel, we show the 68 and 95\% CL contours (red and blue, respectively) both after applying the CMS [10] and ATLAS [11] constraints (dashed and solid lines, respectively) and beforehand (dotted lines). Also shown as open (solid) green stars are the best-fit points found after applying the CMS (ATLAS) constraints in each model (see text), and as green 'snowflakes' the previous best-fit points.

In the region of parameter space of interest to the CMSSM, NUHM1, VCMSSM and mSUGRA, the reach of the LHC for SUSY is largely determined by the gluino mass, $m_{\tilde{g}}$. Accordingly, we display in Fig. 5 the one-parameter $\chi^{2}$ functions for $m_{\tilde{g}}$ relative to the minima in all these models. In each case, we display the new likelihood functions incorporating CMS or ATLAS data as dashed or solid lines, respectively, and those given by the previous fits as dotted lines (see also Figs. 1, 2), setting to zero the value of $\chi^{2}$ at the best fit in each model. In this figure the one-parameter $\chi^{2}$ functions for mSUGRA are unchanged when the LHC data are included but are shown for comparison purposes. 

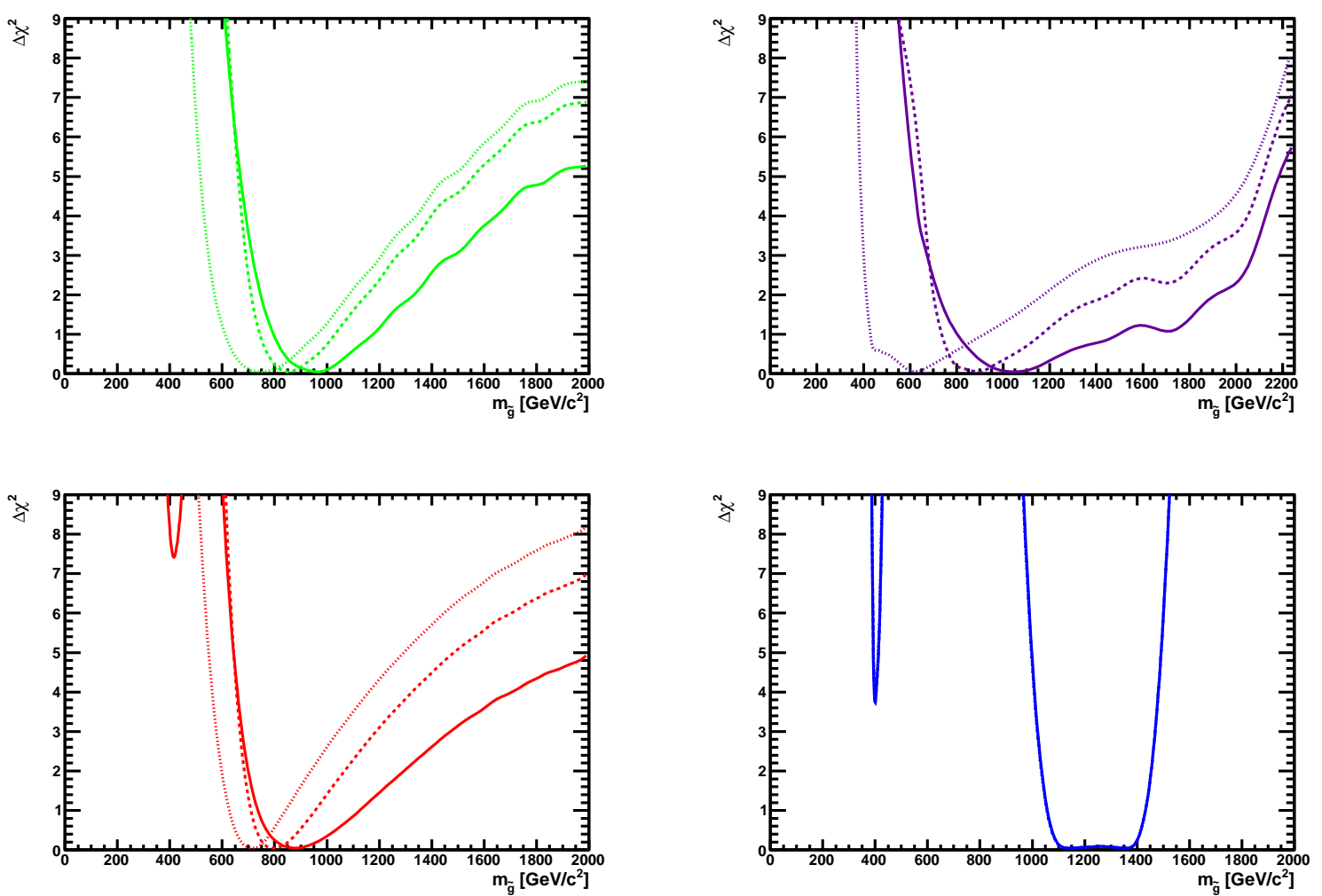

Figure 5: The one-parameter $\chi^{2}$ likelihood functions for the gluino mass $m_{\tilde{g}}$ in the CMSSM (upper left), NUHM1 (top right), VCMSSM (lower left) and MSUGRA (lower right). In each panel, we show the $\chi^{2}$ function including the CMS or ATLAS [10,11] constraints as dashed and solid lines, respectively, and the previous $\chi^{2}$ function as a dotted line.

For each of the CMSSM, NUHM1 and VCMSSM, we see that the side of the likelihood function below the best-fit point is shifted to larger $m_{\tilde{g}}$ by similar amounts $\delta m_{\tilde{g}} \sim 100$ to $200 \mathrm{GeV}$. The best-fit values of $m_{\tilde{g}}$ in the CMSSM, NUHM1 and VCMSSM are now $\sim 900 \mathrm{GeV}$. In mSUGRA the most likely values of $m_{\tilde{g}}$ are unchanged, lying in the range $\sim 1000$ to $\sim 1400 \mathrm{GeV}$, rising steeply outside this interval.

\section{Prediction for $M_{h}$ including LHC data}

One of the main goals of the LHC is the discovery of a Higgs boson, revealing the mechanism of electroweak symmetry breaking. Similarly, a huge effort is put in the current Tevatron analyses for SM and MSSM Higgs boson searches. Accordingly, we display in Fig. 6 the one-parameter $\chi^{2}$ functions for the lightest MSSM Higgs mass $M_{h}$ in the CMSSM, NUHM1, VCMSSM and mSUGRA. In this figure we do not include the direct limits from LEP [18,22] or the Tevatron, so as to display the (lack of) conflict between these limits and the predictions of supersymmetric models. For each model we again display the new likelihood functions incorporating CMS (ATLAS) data as dashed (solid) lines, around the latter indicating the theoretical uncertainty in the calculation of 
$M_{h}$ of $\sim 1.5 \mathrm{GeV}$ by red bands. We also show, as dotted lines without red bands, the pre-LHC predictions for $M_{h}$ (again discarding the LEP constraint.)
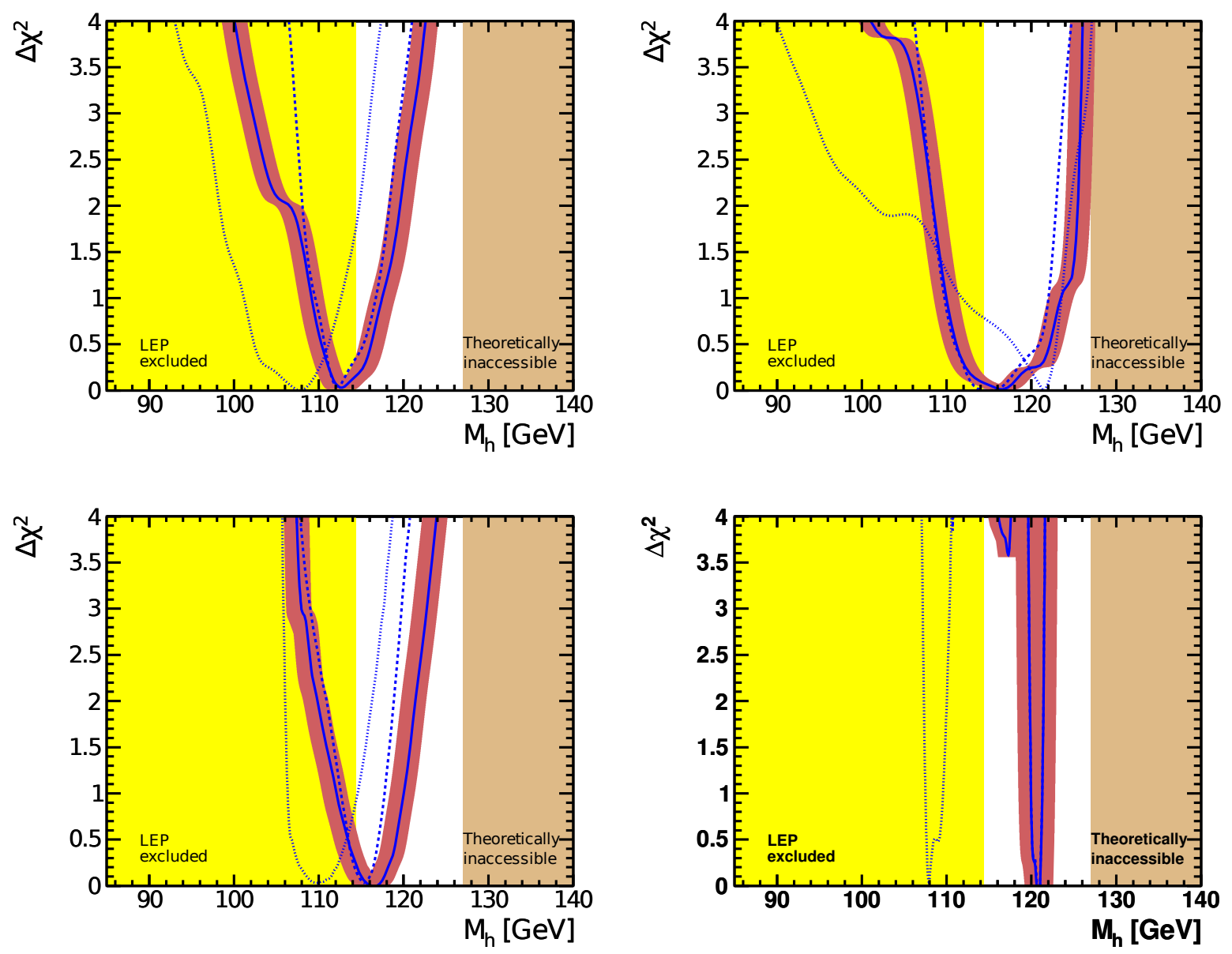

Figure 6: The one-parameter $\chi^{2}$ likelihood functions for the lightest MSSM Higgs mass $M_{h}$ in the CMSSM (upper left), NUHMI (top right), VCMSSM (lower left) and MSUGRA (lower right). In each panel, we show the $\chi^{2}$ functions including the CMS (ATLAS) [10,11] constraints as dashed (solid) lines, the latter with a red band indicating the estimated theoretical uncertainty in the calculation of $M_{h}$ of $\sim 1.5 \mathrm{GeV}$, and the pre-LHC $\chi^{2}$ function is shown as a dotted line.

In the case of the CMSSM, we see that the LHC constraints increases the consistency of the model prediction with the direct LEP limit on $M_{h}$, indicated by the yellow region: the best-fit value can be found at $\sim 112.6 \mathrm{GeV}$ with an estimated theoretical error of $1.5 \mathrm{GeV}$, see also Tab. 1 . In the case of the NUHM1, we see that the main effect of the LHC data is to increase substantially the one-parameter $\chi^{2}$ function at low masses $M_{h}<110 \mathrm{GeV}$, see Sect. 2 for details. In the NUHM1 the LEP constraint is weakened at low $M_{h}$ because the $h Z Z$ coupling may be reduced (which is not possible in the CMSSM, VCMSSM and mSUGRA [19,20]). The LHC data render a large reduction less likely. Now most of the preferred $M_{h}$ region in the NUHM1 is indeed above $\sim 114 \mathrm{GeV}$. In the case of the VCMSSM, the LHC data strongly disfavor a Higgs boson below the LEP limit. In the case of mSUGRA, low $M_{h} \sim 110 \mathrm{GeV}$ would have been favored pre-LHC, but the CMS or ATLAS data push the preferred range to larger $M_{h}$ compatible with the LEP constraint $[18,22]$. 
Thus, including the LHC data, within NUHM1, VCMSSM and mSUGRA the combination of all other experimental constraints naturally evades the LEP Higgs constraints, and no tension between $M_{h}$ and the experimental bounds exists. Within the CMSSM a "tension" smaller than in the SM can be observed.

\section{Acknowledgements}

We thank O. Buchmüller, R. Cavanaugh, D. Colling, A. De Roeck, M. Dolan, J. Ellis, H. Flächer, G. Isidori, K. Olive, S. Rogerson, F. Ronga and G. Weiglein with whom the results presented here have been obtained. We are grateful to the support of the Spanish MICINN's Consolider-Ingenio 2010 Program under grant MultiDark CSD2009-00064. Finally, we thank the organizers of Kruger 2010: Workshop on Discovery Physics at the LHC for the invitation and an inspiring workshop, as well as the elephant for showing up at the conference dinner.

\section{References}

[1] H. P. Nilles, Phys. Rept. 110 (1984) 1.

[2] H. Haber and G. Kane, Phys. Rept. 117 (1985) 75.

[3] R. Barbieri, S. Ferrara and C. A. Savoy, Phys. Lett. B 119 (1982) 343.

[4] O. Buchmueller et al., Phys. Lett. B 657 (2007) 87 [arXiv:0707.3447 [hep-ph]].

[5] O. Buchmueller et al., JHEP 0809 (2008) 117 [arXiv:0808.4128 [hep-ph]].

[6] O. Buchmueller et al., Eur. Phys. J. C 64 (2009) 391 [arXiv:0907.5568 [hep-ph]].

[7] O. Buchmueller et al., Phys. Rev. D 81 (2010) 035009 [arXiv:0912.1036 [hep-ph]].

[8] O. Buchmueller et al., to appear in Eur. Phys. J. C, arXiv:1011.6118 [hep-ph].

[9] O. Buchmueller et al., arXiv:1102.4585 [hep-ph].

[10] V. Khachatryan et al. [CMS Collaboration], arXiv:1101.1628 [hep-ex].

[11] G. Aad et al. [The ATLAS Collaboration], arXiv:1102.2357 [hep-ex].

[12] [The Muon g-2 Collaboration], Phys. Rev. Lett. 92 (2004) 161802. [arXiv:hep-ex/0401008]; G. Bennett et al. [The Muon g-2 Collaboration], Phys. Rev. D 73 (2006) 072003 [arXiv:hep-ex/0602035].

[13] D. Stockinger, J. Phys. G 34 (2007) R45 [arXiv:hep-ph/0609168]; J. Miller, E. de Rafael and B. Roberts, Rept. Prog. Phys. 70 (2007) 795 [arXiv:hep-ph/0703049]; J. Prades, E. de Rafael and A. Vainshtein, arXiv:0901.0306 [hep-ph]; F. Jegerlehner and A. Nyffeler, Phys. Rept. 477, 1 (2009) [arXiv:0902.3360 [hep-ph]]; J. Prades, Acta Phys. Polon. Supp. 3, 75 (2010) [arXiv:0909.2546 [hep-ph]]; T. Teubner, K. Hagiwara, R. Liao, A. D. Martin and D. Nomura, arXiv:1001.5401 [hep-ph].

[14] M. Davier, A. Hoecker, B. Malaescu and Z. Zhang, arXiv:1010.4180 [hep-ph].

[15] F. Jegerlehner and R. Szafron, arXiv:1101.2872 [hep-ph].

[16] LEP Supersymmetry Working Group, lepsusy . web.cern.ch/lepsusy/.

[17] Tevatron Electroweak Working Group and CDF Collaboration and D0 Collaboration, arXiv:1007.3178 [hep-ex]. 
[18] R. Barate et al. [ALEPH, DELPHI, L3, OPAL Collaborations and LEP Working Group for Higgs boson searches], Phys. Lett. B 565 (2003) 61 [arXiv:hep-ex/0306033].

[19] J. R. Ellis, S. Heinemeyer, K. A. Olive and G. Weiglein, Phys. Lett. B 515 (2001) 348 [arXiv:hep-ph/0105061].

[20] S. Ambrosanio, A. Dedes, S. Heinemeyer, S. Su and G. Weiglein, Nucl. Phys. B 624 (2002) 3 [arXiv:hep-ph/0106255].

[21] G. Degrassi, S. Heinemeyer, W. Hollik, P. Slavich and G. Weiglein, Eur. Phys. J. C 28 (2003) 133 [arXiv:hep-ph/0212020].

[22] S. Schael et al. [ALEPH, DELPHI, L3, OPAL Collaborations and LEP Working Group for Higgs boson searches], Eur. Phys. J. C 47 (2006) 547 [arXiv:hep-ex/0602042].

[23] P. Bechtle, O. Brein, S. Heinemeyer, G. Weiglein and K. E. Williams, Comput. Phys. Commun. 181 (2010) 138 [arXiv:0811.4169 [hep-ph]]; arXiv:0909.4664 [hep-ph]; arXiv:1102.1898 [hep-ph]; see www . ippp.dur.ac.uk/HiggsBounds.

[24] See: http://cern.ch/mastercode.

[25] B. C. Allanach, Comput. Phys. Commun. 143 (2002) 305 [arXiv:hep-ph/0104145].

[26] S. Heinemeyer, W. Hollik and G. Weiglein, Eur. Phys. J. C 9 (1999) 343 [arXiv:hep-ph/9812472].

[27] S. Heinemeyer, W. Hollik and G. Weiglein, Comput. Phys. Commun. 124 (2000) 76 [arXiv:hep-ph/9812320]. See www . feynhiggs . de .

[28] M. Frank, T. Hahn, S. Heinemeyer, W. Hollik, H. Rzehak and G. Weiglein, JHEP 0702 (2007) 047 [arXiv:hep-ph/0611326].

[29] T. Moroi, Phys. Rev. D 53 (1996) 6565 [Erratum-ibid. D 56 (1997) 4424] [arXiv:hep-ph/9512396].

[30] G. Degrassi and G. F. Giudice, Phys. Rev. D 58 (1998) 053007 [arXiv:hep-ph/9803384].

[31] S. Heinemeyer, D. Stockinger and G. Weiglein, Nucl. Phys. B 690 (2004) 62 [arXiv:hep-ph/0312264].

[32] S. Heinemeyer, D. Stockinger and G. Weiglein, Nucl. Phys. B 699 (2004) 103 [arXiv:hep-ph/0405255].

[33] G. Isidori and P. Paradisi, Phys. Lett. B 639 (2006) 499 [arXiv:hep-ph/0605012].

[34] G. Isidori, F. Mescia, P. Paradisi and D. Temes, Phys. Rev. D 75 (2007) 115019 [arXiv:hep-ph/0703035], and references therein.

[35] F. Mahmoudi, Comput. Phys. Commun. 178 (2008) 745 [arXiv:0710.2067 [hep-ph]] and arXiv:0808.3144 [hep-ph].

[36] D. Eriksson, F. Mahmoudi and O. Stal, JHEP 0811 (2008) 035 [arXiv:0808.3551 [hep-ph]].

[37] S. Heinemeyer, W. Hollik, D. Stockinger, A. M. Weber and G. Weiglein, JHEP 0608 (2006) 052 [arXiv:hep-ph/0604147].

[38] S. Heinemeyer, W. Hollik, A. M. Weber and G. Weiglein, JHEP 0804 (2008) 039 [arXiv:0710.2972 [hep-ph]].

[39] G. Belanger, F. Boudjema, A. Pukhov and A. Semenov, Comput. Phys. Commun. 176 (2007) 367 [arXiv:hep-ph/0607059].

[40] G. Belanger, F. Boudjema, A. Pukhov and A. Semenov, Comput. Phys. Commun. 149 (2002) 103 [arXiv:hep-ph/0112278]. 
[41] G. Belanger, F. Boudjema, A. Pukhov and A. Semenov, Comput. Phys. Commun. 174 (2006) 577 [arXiv:hep-ph/0405253].

[42] P. Gondolo et al., New Astron. Rev. 49 (2005) 149.

[43] P. Gondolo et al., JCAP 0407 (2004) 008 [arXiv:astro-ph/0406204].

[44] P. Skands et al., JHEP 0407 (2004) 036 [arXiv:hep-ph/0311123].

[45] B. Allanach et al., Comput. Phys. Commun. 180 (2009) 8 [arXiv:0801.0045 [hep-ph]].

[46] G. Aad et al. [The ATLAS Collaboration], arXiv:0901.0512.

[47] G. L. Bayatian et al., CMS Collaboration, J. Phys. G 34 (2007) 995; see: cmsdoc.cern.ch/cms/cpt/tdr/.

[48] F. Gianotti et al., Eur. Phys. J. C 39 (2005) 293 [arXiv:hep-ph/0204087].

[49] TESLA Technical Design Report [TESLA Collaboration], arXiv:hep-ph/0106315; see: tesla.desy.de/new_pages/TDR_CD/start.html.

[50] J. Brau et al. [ILC Collaboration], ILC Reference Design Report Volume 1 - Executive Summary, arXiv:0712.1950 [physics.acc-ph];

G. Aarons et al. [ILC Collaboration], International Linear Collider Reference Design Report Volume

2: Physics at the ILC, arXiv:0709.1893 [hep-ph].

[51] J. Ellis, S. Heinemeyer, K.A. Olive, A.M. Weber, G. Weiglein, JHEP 08 (2007) 083 [arXiv:0706.0652 [hep-ph]]

[52] J. Ellis, T. Hahn, S. Heinemeyer, K. A. Olive and G. Weiglein, JHEP 0710 (2007) 092 [arXiv:0709.0098 [hep-ph]].

[53] M. S. Carena, S. Heinemeyer, C. E. M. Wagner and G. Weiglein, Eur. Phys. J. C 26 (2003) 601 [arXiv:hep-ph/0202167].

[54] M. S. Carena, S. Heinemeyer, C. E. M. Wagner and G. Weiglein, Eur. Phys. J. C 45 (2006) 797 [arXiv:hep-ph/0511023].

[55] S. Gennai, S. Heinemeyer, A. Kalinowski, R. Kinnunen, S. Lehti, A. Nikitenko and G. Weiglein, Eur. Phys. J. C 52 (2007) 383 [arXiv:0704.0619 [hep-ph]].

[56] S. Heinemeyer and G. Weiglein, Nucl. Phys. Proc. Suppl. 205-206 (2010) 283 [arXiv:1007.0206 [hep-ph]].

[57] S. Heinemeyer, arXiv:1009.2678 [hep-ph]. 